\title{
Infections of cats with blood mycoplasmas in various contexts
}

\author{
Dana Lobová ${ }^{1}$, Jarmila Konvalinová2 ${ }^{2}$ Iveta Bedáňová², Zita Filipejová3, \\ Dobromila Molinková ${ }^{1}$
}

\begin{abstract}
University of Veterinary Sciences Brno, ${ }^{1}$ Faculty of Veterinary Medicine, Department of Infectious Diseases and Microbiology, ${ }^{2}$ Faculty of Veterinary Hygiene and Ecology, Department of Animal Protection, Welfare and Ethology, ${ }^{3}$ Faculty of Veterinary Medicine, Small Animal Clinic, Brno, Czech Republic
\end{abstract}

Received April 1, 2020

Accepted May 26, 2021

\begin{abstract}
Haemotropic microorganisms are the most common bacteria that infect erythrocytes and are associated with anaemia of varying severity. The aim of this study was to focus on the occurrence of Mycoplasma haemofelis, Mycoplasma haemominutum, and Mycoplasma turicensis in cats. We followed infected individuals' breeding conditions, age, sex, basic haematological indices, and co-infection with one of the feline retroviruses. A total of 73 cats were investigated. Haemoplasmas were detected by PCR and verified by sequencing. Haematology examination was performed focusing on the number of erythrocytes, haemoglobin concentrations and haematocrit. A subset of 40 cat blood samples was examined by a rapid immunochromatography test to detect retroviruses. The following was found in our study group: M. haemofelis in $12.3 \%$ of individuals, $M$. haemominutum in $35.6 \%$ of individuals and $M$. turicensis in $17.8 \%$ of individuals. A highly significant difference was found between positive evidence of blood mycoplasmas in cats living only at home $(15 \%)$ and in cats with access to the outside $(69.8 \%)$. There was also a highly significant difference in the incidence of mycoplasma in cats over 3 years of age compared to 1-3 years of age and up to 1 year of age. There was no difference in the frequency of infections between the sexes. Blood mycoplasma infection in our group was not accompanied by fundamental changes in the haematological indices and was only reflected by a decrease in haemoglobin values in three cases. In a subset of cats that were also examined for the presence of retroviral infection, mycoplasma infection in blood was confirmed in all five positive cases.
\end{abstract}

Blood pathogens, feline category, PCR, haematology

The haemotropic microorganisms found in cats include a group of bacteria that attack erythrocytes (Messick et al. 1998). They are transmitted by arthropods (Chomel et al. 2009). The three main bacterial pathogens tested include Mycoplasma haemofelis, Mycoplasma haemominutum, and Mycoplasma turicensis. Detection of these pathogens is associated with the development of molecular biological diagnostic methods due to the lack of reliable culture detection.

These mycoplasmas belong to the family Mycoplasmataceae and are referred to as agents of feline infectious anaemia (FIA). Based on the 16S rRNA gene sequence, two important species were identified in cats, Mycoplasma haemofelis, formerly Haemobartonella felis large (Hflg) and Mycoplasma haemominutum, formerly Haemobartonella felis small (Hfsm) (Niemark at al. 2001; Messick 2004). A new species of Mycoplasma turicensis was identified in 2005 (Willi at al. 2005). Their occurrence is global. Mycoplasma haemofelis causes severe macrocytic normochromic anaemia with the appearance of nuclear erythrocytes. Mycoplasma haemominutum causes no or mild haematological abnormalities (Foley et al. 1998). Mycoplasma turicensis may be the cause of haemolytic anaemia in cats (Santos et al. 2009). Blood mycoplasmas can be transmitted by infected blood (blood transfusions) or through lice, fleas, ticks and mosquitoes. Vertical transmission from mother to offspring is also possible. Transmission of these pathogens during fights

Address for correspondence:

MVDr. Dana Lobová, Ph.D.

Department of Infectious Diseases and Microbiology

Faculty of Veterinary Medicine, VetUni Brno

Phone: +420 541562290

Palackého tř. 1946/1, 61242 Brno

http://actavet.vfu.cz/ 
is also likely. This theory is supported by studies showing the presence of haemoplasmic DNA in the saliva and claws of infected cats (Messick 2004). The incidence of blood pathogens of bacterial origin increases in immunosuppressed animals infected with Feline Immunodeficiency Virus (FIV) and Feline Leukaemia Virus (FeLV) (Tasker 2010). The risk group for the occurrence of FIV are uncastrated males older than 6 years (Sykes 2014). A FeLV infection may occur in very young animals. Despite the fact that blood mycoplasmas can cause serious clinical symptoms in their hosts or complicate the care of animals with retroviral infections, there is not yet enough information regarding their occurrence in the Czech Republic.

Taking into account these facts, this study focused on the general determination of the incidence of feline blood pathogens in animals from the Czech Republic that were subjected to veterinary examination, including a haematology analysis focusing on the erythrocyte count, haemoglobin level, and haematocrit. The aim of this study was to obtain information on the occurrence of these bacteria, to monitor their effect on haematological indices in individual cats as well as to draw attention to predisposing factors and focus on retroviral co-infection. The results of the study should increase the awareness of this group of pathogens among the veterinary profession, enrich the spectrum of common diagnostic methods and contribute to the targeted therapy of these infections.

\section{Materials and Methods}

\section{Samples}

We investigated blood samples from a total of 73 cats with different medical histories and different clinical manifestations. A total of 20 cats from the basic group (27\%) came from a purely domestic environment (indoor), 53 cats $(73 \%)$ had contact with the outdoor environment and were probably infested with ectoparasites. There were 23 females $(31.5 \%)$ and 50 males $(68.5 \%)$. The total population was divided into 3 age categories: 7 animals up to 1 year of age $(10 \%), 12$ animals aged $1-3$ years $(16 \%)$ and a total of $54(74 \%)$ animals that were over 3 years old. The results of the basic haematological examination were available in the whole group of monitored cats. In this study, the following values were monitored: the erythrocyte count, the amount of haemoglobin and haematocrit. Blood was collected from the cats by veterinarians in a sterile way into test tubes with anticoagulant (EDTA) during basic veterinary examinations. After the haematological blood testing at the Central Clinical Laboratory, VetUni Brno (Celltac alpha MEK), blood was frozen at $-20^{\circ} \mathrm{C}$.

Positive control strains

Positive control strains of mycoplasma were obtained from Dr. Messick of the Department of Comparative Pathobiology, School of Veterinary Medicine, Purdue University, West Lafayette, Indiana, USA and were designed by Jensen at al. (2001).

\section{Primers}

For the amplification of $M$. haemofelis and $M$. haemominutum, the same primers were used to distinguish both pathogens based on the molecular weight of the resulting PCR products. For detection of $M$. turicensis, primers from Brazil were used (Santos et al. 2009). Primer sequences are shown in Table 1.

Table 1. Primer sequences.

\begin{tabular}{|c|c|c|c|}
\hline Primer name & Primer sequence & Size/bp & Reference \\
\hline & & \multicolumn{2}{|c|}{ (base pair) } \\
\hline M. haemofelis for & 5'ACG AAA GTC TGA TGG AGC AAT A-3' & 170 & Jensen et al. 2001 \\
\hline M. haemofelis rev & 5'ACG CCC AAT AAA TCC GRA-3' & & \\
\hline M. haemominutum for & 5'ACG AAA GTC TGA TGG AGC AAT A-3' & 193 & Jensen et al. 2001 \\
\hline M. haemominutum rev & 5'ACG CCC AAT AAA TCC GRA-3' & & \\
\hline M. turicensis for & 5'-GAAAAA TTT GAT GGT ACC CTC-3' & 488 & Santos at al. 2009 \\
\hline M. turicensis rev & 5 ,-GCC GAA ACA CAA ATC CCG AC-3' & & \\
\hline
\end{tabular}


Detection of M. haemofelis, M. haemominutum, and M. turicensis using PCR

Nucleic acids extraction and PCR: Total DNA was extracted by commercial extraction kits according to the manufacturer's instructions (NucleoSpin Blood DNA, Macherey-Nagel, Düren, Germany). Detection of bacterial DNA was done using Combi PPP Master Mix (Top-Bio, Prague, Czech Republic) by PCR in a total amount of $20 \mu 1$ per sample according to the manufacturer's instructions. Reaction conditions were as follows: $95{ }^{\circ} \mathrm{C} / 2 \mathrm{~min}$, $94{ }^{\circ} \mathrm{C} / 45 \mathrm{~s}, 53{ }^{\circ} \mathrm{C} / 30 \mathrm{~s}, 72{ }^{\circ} \mathrm{C} / 30 \mathrm{~s}, 34 \times$, and final extension $72{ }^{\circ} \mathrm{C} / 5 \mathrm{~min}$ for M. haemofelis. $95^{\circ} \mathrm{C} / 2 \mathrm{~min}, 94^{\circ} \mathrm{C} / 45 \mathrm{~s}$, $54{ }^{\circ} \mathrm{C} / 45 \mathrm{~s}, 72{ }^{\circ} \mathrm{C} / 45 \mathrm{~s}, 36 \times$ and final extension $7 \mathrm{~min}$ for $M$. haemominutum and for M. turicensis. Amplified PCR products were visualized in $2 \%$ agarose gel.

Gel extraction: Gel/PCR DNA Fragments Extraction Kit (Geneaid Biotech Ltd, New Taipei City, Taiwan) was used according to the manufacturer's instructions to extract the amplified DNA from agarose gel. The DNA fragments obtained were stored at $-80^{\circ} \mathrm{C}$.

DNA sequencing of $16 \mathrm{~S}$ rRNA gene of M. haemofelis, M. haemominutum and M. turicensis

The part of the genome which encodes the 16S rRNA gene of feline haemotropic bacteria was sequenced in Macrogen (Amsterdam, Netherlands). DNA sequences were compared with DNA sequences of reference strains from the GenBank database. Sequence analysis was performed by Geneious V 10.2.3. software (Auckland, New Zealand).

Detection of feline retroviruses

The FeLV antigen and FIV antibodies were detected by rapid immunochromatographic test (SNAP FIV/FeLV Combo Test, IDEXX, Westbrook, Maine, USA) in a total of 40 samples of blood.

Statistical analysis

Frequencies of pathogens in infected cats in different living conditions (indoor/outdoor), sex categories, different age groups and the influence of mycoplasmas on selected blood indices (haematocrit, haemoglobin, erythrocyte count) were compared on the basis of chi-square analysis of $r \times c$ and $2 \times 2$ contingency tables (with Yates correction) (Zar 1999). In the case of frequencies lower than five, Fisher's exact test was used instead of chi-square test. A $P$ value $<0.05$ was considered significant.

\section{Results}

\section{Occurrence of blood mycoplasmas}

Of the total of 73 samples examined by PCR and verified by sequencing, some of the blood mycoplasmas or a combination of them were detected in a total of 40 animals $(55 \%)$. Ten animals were infected with multiple species at one time (25\% of all positive cases). The presence of $M$. haemofelis was demonstrated in 9 cases $(22.5 \%$ of all positive cases), $M$. heamominutum in 26 cases (65\% of all positive cases) and M. turicensis in 13 cases (32.5\% of all positive cases) (Table 2 ).

Table 2. Incidence of mycoplasma species.

\begin{tabular}{lcc}
\hline Type of haemoplasma & $\begin{array}{c}\text { Number of positive } \\
\text { animals from a total of } 73\end{array}$ & $\begin{array}{c}\text { Percentage of proof of species } \\
\text { from all } 40 \text { positive cases }\end{array}$ \\
\hline M. heamofelis & $9(12.3 \%)$ & $22.5 \%$ \\
M. heamominutum & $26(35.6 \%)$ & $65 \%$ \\
M. turicensis & $13(17.8 \%)$ & $32.5 \%$ \\
\hline
\end{tabular}

Altogether, 20 cats from the basic group (27\%) came from a purely domestic environment, 53 cats $(73 \%)$ had contact with the outdoor environment (Table 3$)$.

In cats kept at home, the presence of blood mycoplasmas was confirmed in 3 cases only $(15 \%)$, of which two were co-infections with different types of haemoplasmas (Table 3$)$. Cats with access to the outdoors were positive in 37 cases $(69.8 \%)$ of which eight were co-infections with various types of haemoplasmas (Table 3 ). The difference in positivity rates between domestic and outdoor cats was determined to be highly significant $(P<0.01)$ in favour of cats in contact with the outdoor environment. 
Table 3. Comparison of occurrence of blood mycoplasma between indoor and outdoor.

\begin{tabular}{llrr}
\hline & & & Housing \\
Total & & Indoor & Outdoor \\
\cline { 2 - 4 } Monoinfections & MHF & 20 & 53 \\
& MHM & 1 & 6 \\
& MT & 0 & 17 \\
Co-infections & MHF + MHM & 1 & 6 \\
& MHM + MT & 1 & 1 \\
& MHF + MT & 0 & 6 \\
\hline
\end{tabular}

MHF - M. haemofelis; MHM - M. haemominutum; MT - M. turicensis

Of a total of 73 cats, $23(31.5 \%)$ were females and $50(68.5 \%)$ males. Of the animals studied, 14 females $(61 \%)$ were positive for the presence of mycoplasmas, of which three infections $(21 \%)$ were mixed. Of the 50 males, the presence of haemoplasmas was demonstrated in 25 cases $(50 \%)$, of which seven infections $(14 \%)$ were co-infections (Table 4$)$. There was no significant difference in the frequency of mycoplasma $(P>0.05)$ between females and males. A total of 73 cats were further divided into 3 age categories (Table 5):

Table 4. Comparison of mycoplasmas between sexes.

\begin{tabular}{llrr}
\hline \multirow{2}{*}{ Total } & & Female & Male \\
\cline { 3 - 4 } Monoinfections & MHF & 23 & 50 \\
& MHM & 0 & 6 \\
& MT & 7 & 10 \\
\hline \multirow{3}{*}{ Co-infections } & MHF + MHM & 4 & 2 \\
& MHM + MT & 1 & 1 \\
& MHF + MT & 2 & 5 \\
\hline
\end{tabular}

MHF - M. haemofelis; MHM - M. haemominutum; MT - M. turicensis

Table 5. Comparison of mycoplasma incidence in terms of age.

\begin{tabular}{llccr}
\hline \multirow{2}{*}{ Total } & & Up to 1 year & $1-3$ years & over 3 years \\
\cline { 3 - 5 } Monoinfections & MHF & 7 & 12 & 54 \\
& MHM & 0 & 2 & 5 \\
& MT & 0 & 2 & 15 \\
Co-infections & MHF + MHM & 3 & 1 & 3 \\
& MHM + MT & 0 & 1 & 1 \\
& MHF + MT & 1 & 3 & 3 \\
\hline
\end{tabular}

MHF - M. haemofelis; MHM - M. haemominutum; MT - M. turicensis

In the youngest category up to 1 year, 4 animals were positive $(57 \%)$, of which 1 was a mixed infection $(25 \%)$. There were $9(75 \%)$ positive animals in the $1-3$ years category, of which 4 were positive for more than one haemoplasma (44\%). In the category older than 3 years, positivity was found to be $52 \%$ (28 individuals); in five cases it was a co-infection by several species $(18 \%)$. 
For small numbers of individuals in the category up to 1 year and in the category 1-3 years, the results could not be objectively evaluated statistically.

\section{Influence of mycoplasmas on selected blood indices}

The erythrocyte, haemoglobin and haematocrit values were assessed for changes that might indicate anaemia. Tables 6,7 , and 8 show samples with haematocrit at the lower limit of the reference values. The reference ranges of these haematological indices are the following: haematocrit: $0.22-0.38$ 1/1; haemoglobin: $79-148 \mathrm{~g} / \mathrm{l}$; erythrocyte count: 4.9-9.8 × 10 $12 / 1$ (Doubek et al. 2014). We focused on haematocrit values of $0.31 / 1$ and lower.

Table 6. Blood counts in M. haemofelis positive cats.

\begin{tabular}{lccc}
\hline Environment, sex, age, disease & Erythrocytes $\left(10^{12} / 1\right)$ & Haemoglobin $(\mathrm{g} / \mathrm{l})$ & Haematocrit $(1 / 1)$ \\
\hline Outdoor, male, 5 years, periodontitis & 7.59 & 101 & 0.3 \\
Outdoor, male, 4 years, chronic hepatitis & 5.8 & 77 & 0.23 \\
Outdoor, female, 1.5 years, polytrauma & 8.17 & 104 & 0.3 \\
Outdoor, male, 10 years, apathy & 6.68 & 93 & 0.26 \\
Reference values & $4.9-9.8$ & $79-148$ & $0.22-0.38$ \\
\hline
\end{tabular}

Table 7. Blood count values in M. haemominutum positive cats.

\begin{tabular}{lccc}
\hline Environment, sex, age, disease & Erythrocytes $\left(10^{12} / 1\right)$ & Haemoglobin $(\mathrm{g} / \mathrm{l})$ & Haematocrit $(\mathrm{l} / \mathrm{l})$ \\
\hline Outdoor, female, 10 years, trauma & 7.25 & 90 & 0.3 \\
Outdoor, male, 2 years, polytrauma, “no. 25” & 4.73 & 75 & 0.22 \\
Outdoor, male, 7 years, weight loss & 6.64 & 97 & 0.3 \\
Outdoor, female, 5 years, weight loss & 8.13 & 100 & 0.3 \\
Outdoor, female, 15 years, chronic kidney disease & 6.96 & 93 & 0.28 \\
Outdoor, female, 1.5 years, polytrauma & 8.17 & 104 & 0.3 \\
Indoor, male, 12 years, septic inflammation & 6.08 & 95 & 0.3 \\
Reference values & $4.9-9.8$ & $79-148$ & $0.22-0.38$ \\
\hline
\end{tabular}

Table 8. Blood count values in M. turicensis positive cats.

\begin{tabular}{lccc}
\hline Environment, sex, age, disease & Erythrocytes $\left(10^{12} / 1\right)$ & Haemoglobin $(\mathrm{g} / \mathrm{l})$ & Haematocrit $(1 / 1)$ \\
\hline Outdoor, female, 3 years, skin lesions, gingivitis & 5.94 & 62 & 0.19 \\
Outdoor, male, 2 years, polytrauma, "no. 25" & 4.73 & 75 & 0.22 \\
Outdoor, female, 1 year, trauma & 6.93 & 85 & 0.25 \\
Outdoor, male, 7 years, apathy & 6.18 & 93 & 0.29 \\
Outdoor, male, 7 years, weight loss & 6.64 & 97 & 0.3 \\
Outdoor, female, 5 years, weight loss & 8.13 & 100 & 0.3 \\
Outdoor, male, 5 years, periodontitis & 7.59 & 101 & 0.3 \\
Reference values & $4.9-9.8$ & $79-148$ & $0.22-0.38$ \\
\hline
\end{tabular}

Of the total of 9 samples positive for $M$. heamofelis, 4 animals (44\%) had reduced haematocrit levels. Of the 26 samples positive for the presence of $M$. heamominutum, 7 animals $(23 \%)$ had reduced haematocrit levels. Of the total of 13 samples positive for M. turicensis, 7 animals (44\%) had reduced haematocrit levels. The differences in haematocrit values of $M$. heamofelis, $M$. heamominutum and $M$. turicensis were not significant $(P>0.05)$. 
There was a non-significant difference between the individual blood counts in the three types of blood mycoplasma $(P>0.05)$. In three samples, lower haemoglobin values were found compared to the reference values. Sample no. 25 (male, 2 years old, outdoor, polytrauma) infected with $M$. heamominutum + M. turicensis showed a decrease in all three blood indices.

\section{Examination for the presence of FeLV and FIV}

A total of 40 cat blood samples that were previously screened for blood mycoplasmas by PCR were screened in the laboratory using SNAP test. The test demonstrates the presence of antibodies against FIV and the FeLV antigen in the blood of cats.

Of all 40 analysed samples, 5 cats infected with retroviruses $(13 \%)$ were identified. The FeLV antigen was detected in 2 animals, antibodies against the FIV virus were detected in 2 animals and one animal was co-infected with both viruses (it was a chronically ill 8-year-old cat in which $M$. haemofelis was also detected). All five positive samples were also positive for blood mycoplasmas. Two samples showed co-infection of FeLV and $M$. haemominutum, two samples were positive for FIV and M. haemominutum and M. turicensis. One patient was positive for both retroviruses and M. turicensis (Table 9).

Table 9. Co-infection of blood mycoplasmas with retroviruses.

\begin{tabular}{lccc}
\hline Retroviruses/blood mycoplasmas & MHM + MT & MHM & MT \\
\hline FeLV & 2 & 2 & \\
FIV & & & 1 \\
FeLV + FIV & & & \\
\hline
\end{tabular}

MHM - M. haemominutum; MT - M. turicensis

\section{Discussion}

In clinical practice, we regularly encounter cases of undiagnosed transient anaemia, inappetence or poor fitness. Methods of molecular biology are of utmost importance in the diagnosis of feline diseases caused by blood mycoplasmas. Cultivation of feline blood to diagnose blood mycoplasmas is not routinely performed, as described by Greene (2006). Microscopic analysis of bacteria adhering to erythrocytes is possible, but the finding is not completely reliable because of the presence of various artefacts. We focused on the occurrence of blood mycoplasmas in feline blood using PCR and sequencing of the $16 \mathrm{~S}$ rRNA gene region.

The prevalence of blood mycoplasmas reported in our monoinfection or co-infection study of 40 cats $(55 \%)$ is much higher than in other European countries. This result may be due to different selections of cats and their total number in the research. Our study focused on the prevalence in cats, especially of outdoor type and higher age categories, with the predominance of cats over three years of age that had previously been infested with ectoparasites. Of the three mycoplasma species identified, M. haemofelis, M. haemominutum, and $M$. turicensis in the population of our cats, the prevalence of M. haemominutum in 26 animals was followed by $M$. turicensis in 13 animals, while the least frequently occurring mycoplasma was $M$. heamofelis in 9 animals. Blood mycoplasmas occur worldwide. A study of mycoplasma in cats in England gave the following results: $16.9 \%$ were positive for M. haemominutum, 1.4\% were positive for M. haemofelis (Tasker et al. 2003). The following results were found in studies in Greece: $10.3 \%$ were positive for $M$. haemominutum and $7.2 \%$ were positive for M. haemofelis. No occurrence of M. turicensis in feline blood was observed (Maher et al. 2010). The overall incidence of blood mycoplasmas in cats in 
southern Germany was only $9.4 \%$ in a similar study from 2016 (Bergmann et al. 2017). A Swiss study observed the prevalence of blood mycoplasma in a cat population using quantitative PCR in clinically healthy and diseased individuals. In contrast to our results, the authors reported a lower prevalence in both healthy and sick cats. Mycoplasma haemominutum was detected in $7 \%$ of healthy animals and in $8.7 \%$ of sick animals (anaemia), M. haemofelis in $2.3 \%$ and $0.2 \%$ of healthy and sick animals, respectively, and M. turicensis in $1.1 \%$ of sick cats only (Willi et al. 2006). In Italy, the prevalence of $M$. haemofelis was $4 \%$, M. haemominutum $12.3 \%$, both lower than in the Czech Republic. Half of the infected cats from the group were co-infected with several species of mycoplasma. The predisposing factor of feline infection was higher age and presence of FIV (Ravagnan et al. 2017). Cats infected with $M$. haemominutum usually display no clinical signs. On the other hand, $M$. haemofelis infection is usually manifested by anaemia and the resulting clinical symptoms.

There were a total of 20 indoor and 53 outdoor animals in our group of cats. Positive samples for mycoplasma came from 3 (15\%) indoor cats and from $37(69.8 \%)$ outdoor cats. Outdoor cats have a high probability of transmission due to fighting. Vectors such as fleas, lice and ticks are also known to be involved in the transmission of this infection. Three positive results in cats from indoor environment can be explained by the fact that owners may have reported the cat's current living conditions as indoor but the cat could have come into contact with the infection before it was under the care of the current owners. Another means of transmission may be if there is another animal (such as a dog, or another cat) living in the household together with the cat, that might have carried the vectors (fleas, ticks) transmitting infections indoors. A significant difference was found in the incidence of mycoplasma between indoor and outdoor environments. The most represented was $M$. heamominutum at the ratio of 1 indoor cat and 17 outdoor cats. These results are consistent with Danish experiments in which a significant difference was found between domestic outdoor cats and isolated domestic indoor cats (Rosenquist et al. 2016).

No significant differences were found in the incidence of mycoplasma in terms of sex. The mild differences between the sexes may be due to the aggressive behaviour of uncastrated males and their frequent rivalry and territorial fights, leading to the transmission of infections through scratches and bites. To reduce the number of positive cases, castration is recommended as it reduces aggressive behaviour towards other individuals. The biggest difference between the sexes was observed in $M$. heamofelis at a ratio of 8 positive reactions in males and 1 positive reaction in females. A study on the occurrence of mycoplasma in cats in southern Brazil has shown a highly significant difference between sexes at $P<0.01$, with the predominant number of positive males (Santos et al. 2014). The outcome of the study is not consistent with ours, namely that blood mycoplasma infection occurs primarily in the male sex. Furthermore, an effect of age on the occurrence of blood mycoplasmas was observed. Most positive mycoplasma samples observed were in the category older than 3 years in $28(52 \%)$ animals. A combination of several mycoplasmas in a single sample was observed in 10 individuals. Of these, 7 were simultaneously positive for $M$. haemominutum and $M$. turicensis, 2 were positive for $M$. heamominutum and $M$. haemofelis, and 1 was positive for $M$. haemofelis and $M$. turicensis. In the study from southern Brazil, a significant difference of $P<0.05$ was demonstrated in mycoplasma occurrence with regard to age of. In the study, mycoplasmas were most prevalent in cats over 3 years of age, as was the case in our study (Santos et al. 2014).

In 40 mycoplasma-positive cats, the blood count obtained from the laboratory of the Department of Dog and Cat Diseases at VetUni Brno was also evaluated. Based on the haematological examination, the following categories were assessed: haematocrit value, haemoglobin level, and erythrocyte count in feline blood. Haemoplasmas causing various types of anaemia may be responsible for the decreased values of the aforementioned three 
indices (Tasker at al. 2009). Blood count was monitored in 40 positive cats, of which $18(45 \%)$ displayed reduced haematocrit levels.

The differences in haematocrit values in samples infected with $M$. heamofelis, $M$. heamominutum and $M$. turicensis were not significant. The difference in the erythrocyte count in $M$. heamofelis was not significantly different from $M$. heamominutum and M. turicensis. It was slightly decreased in one cat (no. 25). The mycoplasma species did not differ from each other in haemoglobin values either. These were reduced only in three cats, two infected with $M$. turicensis, and one infected with $M$. haemofelis. There are many adaptable mechanisms in a healthy animal, keeping the basic physiologic indicators at optimal levels in cats with quite a good health status (Doubek et al. 2014).

However, if haemotropic bacteria co-infect with other pathogens, they may potentiate the development of clinical symptoms. Cats infected with FIV or FeLV suffer much longer infections with blood mycoplasma due to immunosuppression (Tasker 2010). Of the two FeLV-positive cats, both were co-infected with M. haemominutum and one with FIV and $M$. turicensis. Two of the FIV positive cats were simultaneously infected with M. haemominutum and M. turicensis (Table 9).

In conclusion, this study summarizes observations and laboratory tests on blood mycoplasmas in cats. In the Czech Republic, laboratories only rarely perform blood mycoplasma tests using the PCR method, while blood smear microscopy is not sufficiently sensitive. This study offers a broader context for assessment and opens up the possibility of verifying clinical conclusions in the laboratory. Mycoplasmosis occurs at any age in cats, and up to a third of untreated animals die from severe anaemia (Sykes 2010). Therefore, it is important to pay particular attention to the occurrence of these pathogens and to focus especially on cats weakened by retroviral infections. The monitoring of animals divided in such manner was a "pilot study". From these unrepeatable results, consequences will be tracked down, and based on these results, exact groups of animals will be set up for another study.

The contribution of this study is in making available to the veterinary profession the diagnostic methods we have established, with optional testing of blood samples of suspect cats. Another future goal will be to introduce real time multiplex PCR (qPCR) to identify all major types of blood pathogens simultaneously. Our study has demonstrated a relatively high incidence of mycoplasmas in cat populations in the Czech Republic. Therefore, our focus going forward should be on the occurrence of these pathogens, especially in cats that show symptoms of anaemia, as well as on better evaluation of their impact on health, including the blood count.

\section{Acknowledgement}

This work was supported by the grant IGA VFU Brno 108/2018/FVL.

\section{References}

Bergmann M, Englert T, Stuetzer B, Hawley JR, Lappin MR, Hartmann K 2017: Risk factors of different hemoplasma species infections in cats. BMC Vet Res 13: 52

Doubek J, Rehakova K, Uhrikova I, Slosarkova S, Doubek R, Matalova E, Bouda J, Petrasova J, Tomenendalova J, Sucman E, Toman M, Faldyna M, Ondrackova P, Matiasovic J, Halouzka R 2014: Základy laboratorní diagnostiky neinfekčních nemocí (in Czech, Basics of Laboratory Diagnostic Methods of Non-infectious Diseases). Astron studio, Prague, Czech Republic, pp. 40-70

Foley JE, Harris S, Poland A, Chomel B, Pedersen NC 1998: Molecular, clinical, and pathologic comparison of two distinct strains of Haemobartonella felis in domestic cats. Am J Vet Res 59: 1581-1588

Greene E 2006: Hemothropic mycoplasmosis (Haemobartonellosis). In: Infectious Diseases of the Dog and Cat. $3^{\text {rd }}$ edn. Elsevier Saunders, St. Louis, USA, pp. 252-260

Chomel BB, Boulouis HJ, Breitschwerdt EB, Kasten RW, Vayssier-Taussat M, Birtles RJ, Koehler JE, Dehio C 2009: Ecological fitness and strategies of adaptation of Bartonella species to their host and vectors. Vet Res 40: $1-22$ 
Jensen WA, Lappin MR, Kamkar S, Reagan WJ 2001: Use of a polymerase chain reaction assay to detect and differentiate two strains of Haemobartonella felis in naturally infected cats. In Am J Vet Res 62: 604-608

Maher IE, Tasker S, Polizopoulou Z, Dasopoulou A, Egan K, Helps CHR, Papasouliotis K 2010: Polymerase chain reaction survey of feline haemoplasma infections in Greece. J Feline Med Surg 12: 601-605

Messick JB, Berent LM, Cooper SK 1998: Development and evaluation of a PCR-based assay for detection of Haemobartonella felis in cats and differentiation of $H$. felis from related bacteria by restriction fragment length polymorphism analysis. J Clin Microbiol 36: 462-466

Messick JB 2004: Hemotrophic mycoplasmas (hemoplasmas): a review and new insights into pathogenic potential. Vet Clin Pathol 33: 2-13

Niemark H, Johansson KE, Rikihisa Y, Tully G 2001: Proposal to transfer some members of the Genera Haemobartonella and Eperythrozoon to the genus Mycoplasma with descriptions of 'Candidatus Mycoplasma haemofelis,' 'Candidatus Mycoplasma haemomuris,' 'Candidatus Mycoplasma haemosuis' and 'Candidatus Mycoplasma wenyonii’. Int J Syst Evol Microbiol 51: 891-899

Ravagnan S, Carli E, Piseddu E, Da Rold G, Porcellato E, Zanardello C, Carminato A, Vascellari M, Capelli G 2017: Prevalence and molecular characterization of canine and feline hemotropic mycoplasmas (hemoplasmas) in northern Italy. Parasit Vectors 10: 132

Rosenquist MB, Meilstrup AKH, Larsen J, Olsen JE, Jensen AL, Thomsen LE 2016: Prevalence of feline haemoplasma in cats in Denmark. Acta Vet Scand 58: 78

Tasker S, Helps CHR, Day MJ, Gruffydd-Jones TJ, Harbour DA 2003: Use of real-time PCR to detect and quantify Mycoplasma haemofelis and Candidatus Mycoplasma haemominutum DNA. J Clin Microbiol 41: 439-441

Tasker S, Peters IR, Papasouliotis K, Cue SM, Willi B, Hofmann-Lehmann R, Gruffydd-Jones TJ, Knowles TG, Day MJ, Helps CR 2009: Description of outcomes of experimental infection with feline haemoplasmas: copy numbers, haematology, Coombs' testing and blood glucose concentrations. Vet Microbiol 139: 323-332

Tasker S 2010: Hemotropic mycoplasmas: What's their real significance in cats? J Feline Med Surg 12: 369-381

Santos AP, Messick JB, Biondo AW, Oliveira ST, Pedralli V, Lasta CS 2009: Design, optimization, and application of a conventional PCR assay with an internal control for detection of 'Candidatus Mycoplasma turicensis' $16 \mathrm{~S}$ rDNA in domestic cats from Brazil. Vet Clin Pathol 38: 443-452

Santos AP, Conrado FO, Messick JB, Biondo AW, de Oliveira ST, Guimaraes AM, do Nascimento NC, Pedralli V, Lasta CS, Diaz González FH 2014: Hemoplasma prevalence and hematological abnormalities associated with infection in three different cat populations from Southern Brazil. Rev Bras Parasitol Vet 24: 428-434

Sykes JE 2010: Feline hemotropic mycoplasmas. J Vet Crit Care (San Antonio) 20: 62-69

Sykes JE 2014: Canine and feline infectious diseases. St. Louis, Mo: Elsevier/Saunders, ISBN 978-1-4377-0795-3

Willi B, Boretti FS, Cattori V, Tasker S, Meli ML, Reusch C, Lutz H, Hofmann-Lehmann R 2005: Identification, molecular characterization, and experimental transmission of a new hemoplasma isolate from a cat with hemolytic anemia in Switzerland. J Clin Microbiol 43: 2581-2585

Willi B, Boretti FS, Baumgartner C, Tasker S, Wenger B, Cattori V, Meli ML, Reusch C, Lutz H, HofmannLehmann R 2006: Prevalence, risk factor analysis, and follow up of infections caused by three feline hemoplasma species in cats in Switzerland. J Clin Microbiol 44: 961-969

Zar JH 1999: Biostatistical Analysis. Prentice-Hall, Inc., New Jersey, USA, 663 p. 